\title{
AIP1 mediates TNF- $\alpha$-induced ASK1 activation by facilitating dissociation of ASK1 from its inhibitor 14-3-3
}

\author{
Rong Zhang, ${ }^{1}$ Xiangrong He, ${ }^{1}$ Weimin Liu, ${ }^{1}$ Meng Lu, ${ }^{1}$ Jer-Tsong Hsieh, ${ }^{2}$ and Wang Min ${ }^{1}$ \\ ${ }^{1}$ Center for Cardiovascular Research, University of Rochester Medical Center, Rochester, New York, USA \\ ${ }^{2}$ Department of Urology, University of Texas, Southwestern Medical Center, Dallas, Texas, USA
}

\begin{abstract}
TNF- $\alpha$ activates ASK1 in part by dissociating 14-3-3 from apoptosis signal-regulating kinase 1 (ASK1). In the present study, we identified a novel Ras GTPase-activating protein (Ras-GAP) as an ASK1-interacting protein (AIP1). AIP1 binds to the C-terminal domain of ASK1 via a lysine-rich cluster within the $\mathrm{N}$-terminal $\mathrm{C} 2$ domain. AIP1 exists in a closed form through an intramolecular interaction between the $\mathrm{N}$-terminus and the C-terminus, and TNF- $\alpha$ induces unfolding of AIP1 leading to association of AIP1 with ASK1. Thus, the N-terminus of AIP1 containing the C2 and GAP domains constitutively binds to ASK1 and facilitates the release of 14-3-3 from ASK1. In contrast to 14-3-3, AIP1 binds preferentially to dephosphorylated ASK1. Recruited AIP1 enhances ASK1-induced JNK activation, and the ASK1 binding and the GAP activity of AIP1 are critical for AIP1-enhanced ASK1 activation. Furthermore, TNF-induced ASK1/JNK activation is significantly blunted in cells where AIP1 is knocked down by RNA interference. These data suggest that AIP1 mediates TNF- $\alpha$-induced ASK1 activation by facilitating dissociation of inhibitor 14-3-3 from
\end{abstract} ASK1, a novel mechanism by which TNF- $\alpha$ activates ASK1.

J. Clin. Invest. 111:1933-1943 (2003). doi:10.1172/JCI200317790.

\section{Introduction}

Apoptosis signal-regulating kinase 1 (ASK1), a member of the MAPK kinase kinase (MAP3K) family, is an upstream activator of JNK and p38 MAPK signaling cascades (1). ASK1 can be activated in response to diverse stress and apoptotic stimuli, including proinflammatory mediators such as TNF- $\alpha$ and reactive oxygen species $(2,3)$. Activation of ASK1 triggers various biological responses such as apoptosis, inflammation, differentiation, and survival in different cell types (4-7). Recent studies from ASK1-deficient mice indicate that ASK1 is critical for TNF- $\alpha$ - and reactive oxygen species-induced apoptosis signaling (8). ASK1 is a $170-\mathrm{kDa}$ protein that functionally is composed of an inhibitory $\mathrm{N}$-terminal domain, an internal kinase domain, and a C-terminal regulatory domain. The C-terminal domain of ASK1 binds to the TRAF

Received for publication January 7, 2003, and accepted in revised form March 11, 2003.

Address correspondence to: Wang Min, Center for Cardiovascular Research, University of Rochester Medical Center, 601 Elmwood Avenue, Box 679, Rochester, New York 14642, USA. Phone: (716) 273-1499; Fax: (716) 275-9895;

E-mail: wang.min@rochester.edu.

Conflict of interest: The authors have declared that no conflict of interest exists.

Nonstandard abbreviations used: apoptosis signal-regulating kinase 1 (ASK1); thioredoxin (Trx); endothelial cell (EC); ASK1interacting protein (AIP1); phosphoserine-967 (pSer-967); human umbilical vein endothelial cell (HUVEC); bovine aortic endothelial cell (BAEC); short hairpin RNA (shRNA); Ras GTPase-activating protein (Ras-GAP); pleckstrin homology (PH); PKC-conserved region 2 (C2); alkaline phosphatase (PPase). domain; this association is required for ASK1 activation by TRAF2 and TRAF6 $(3,9)$. Several cellular factors including thioredoxin (Trx), glutaredoxin, and 14-3-3 have been reported to inhibit ASK1 activity. The cellular redox sensors Trx and glutaredoxin in reduced form bind to ASK1 and block cytokine- and reactive oxygen species-induced ASK1 activation $(6,7,10-12)$. 14-3-3, a phosphoserine-binding molecule, binds to ASK1 specifically via Ser-967 of ASK1 and has been reported to inhibit ASK1-induced apoptosis $(6,13)$.

The mechanism by which stress stimuli activate ASK1 is not clear. It has been shown that TNF- $\alpha$ activates ASK1 in part by dissociating ASK1 from its inhibitors Trx and 14-3-3. Trx binds to ASK1 in a reduced form via either one of the two critical Cys residues Cys-32 and Cys-35 of Trx. We have recently shown that association of Trx with ASK1 induces ASK1 ubiquitination and degradation, leading to inhibition of ASK1 activity (7). TNF- $\alpha$ dissociates Trx from ASK1 by generating intracellular reactive oxygen species to oxidize $\operatorname{Trx}(7,10)$. The role of $14-3-3$ in regulation of TNF- $\alpha$-induced ASK1 activation in endothelial cells (ECs) has also been demonstrated. We have previously shown that TNF- $\alpha$ activates ASK1 by dissociating it from the ASK1-14-3-3 complex (6). In contrast, laminar flow in ECs inhibits TNF- $\alpha-$ induced ASK1 and JNK activation by preventing the release of ASK1 from 14-3-3 (6). However, the mechanism by which TNF- $\alpha$ dissociates 14-3-3 from ASK1 is not understood.

In the present study, we show that a novel ASK1-interacting protein (AIP1) associates with ASK1 in response 
to TNF- $\alpha$. AIP1 binds to a sequence surrounding the 14-3-3 binding site (phosphoserine-967, or pSer-967) on ASK1, but binds preferentially to a dephosphorylated active form of ASK1. More importantly, AIP1 induces dissociation of 14-3-3 from ASK1, leading to enhanced ASK1 activity. We propose that AIP1 mediates TNF- $\alpha-$ induced ASK1 activation by facilitating dissociation of 14-3-3, a novel mechanism for ASK1 activation.

\section{Methods}

Plasmid construction. Expression plasmids for wild-type ASK1 (ASK1-WT) and deletion of the N-terminal domain (ASK1- $\Delta \mathrm{N}$ ) were described previously $(6,7)$. For the yeast expression plasmid, ASK1- $\Delta \mathrm{N}$ was amplified by PCR using a $5^{\prime}$ primer with an NdeI site and a $3^{\prime}$ primer with a SalI site. The PCR product was inserted into the NdeI and SalI sites of the expression vector pGBKT7 (Clontech Laboratories Inc., Palo Alto, California, USA) to generate pGBKT-ASK1- $\Delta \mathrm{N}$, in which ASK1- $\Delta \mathrm{N}$ was fused in-frame with the DNA binding domain of yeast transcriptional activator GAL4. The full-length human AIP1 cDNA (AF9q34, GenBank accession no. AY032952) $(14,15)$ was amplified by PCR from a human EC CDNA library using a $5^{\prime}$ primer with a HindIII site and a $3^{\prime}$ primer with an EcoRI site. The PCR product was inserted into the mammalian expression FLAG vector to generate AIP1-F. Similar expression constructs for various AIP1 truncated forms (AIP1-N, -C, -PHC2, and -PH) were also produced. The AIP1 mutants (AIP1-KA1, -KA2, -KA1/2, and -R289L) were constructed using the QuikChange site-directed mutagenesis kit (Stratagene, La Jolla, California, USA) according to the manufacturer's protocol.

Yeast two-bybrid screening. ASK1- $\Delta \mathrm{N}$ bait was used to screen a pretransformed human heart cDNA library (Clontech Laboratories Inc.). Yeast two-hybrid screening was performed according to the instructions of the manufacturer (Clontech Laboratories Inc.). In brief, the yeast strain AH109 harboring pAS-ASK1- $\Delta \mathrm{N}$ was mated with Y190 harboring a human heart cDNA library. Mating zygotes were selected on synthetic dropout agar plates lacking Trp, Leu, His, and Ade (QDO plates). Yeast colonies were transferred onto a nylon membrane and processed using a $\beta$-galactosidase filter assay. Plasmids from positive colonies were isolated and retransformed into the yeast strain Y190 with either PAS2.1 or PAS-ASK1- $\triangle \mathrm{N}$ to confirm that growth on QDO and activity of $\beta$-galactosidase was ASK1- $\Delta \mathrm{N}-$ dependent. The cDNA inserts from true positive clones were subjected to DNA sequencing with a dye terminator cycle sequencing kit (University of Rochester core facility).

Cells and cytokines. Human umbilical vein ECs (HUVECs) and bovine aortic ECs (BAECs) were purchased from Clonetics Corp. (San Diego, California, USA). Human recombinant TNF- $\alpha$ was from R\&D Systems Inc. (Minneapolis, Minnesota, USA) and was used at $10 \mathrm{ng} / \mathrm{ml}$.

Generation of a polyclonal antibody against the N-terminal AIP1. A rabbit polyclonal antibody was generated by immunizing rabbits with GST-AIP1-PH protein through Cocalico Biologicals Inc. (Reamstown, Pennsylvania, USA).

JNK and ASK1 kinase assays. The JNK assay was performed as described previously $(6,7)$ using GST-c-Jun 1-80 containing the $\mathrm{N}$-terminal 80 amino acid residues of c- using Jun fusion protein as a substrate. The ASK1 assay was performed using GST-MKK4 as a substrate.

Transfection and reporter assay. Transfection of HUVECs was performed by the DEAE-dextran method as described previously $(6,7)$. BAECs were transfected by Lipofectamine 2000 (Invitrogen Corp., San Diego, California, USA). Luciferase activity followed by renilla activity was measured twice in duplicate using a Berthold luminometer (EG\&G Wallac, Gaithersburg, Maryland, USA). All data were normalized as relative luciferase light units/renilla unit.

Immunoprecipitation and immunoblotting. HUVECs or BAECs after various treatments were washed twice with cold PBS and lysed in $1.5 \mathrm{ml}$ of cold lysis buffer (50 mM Tris- $\mathrm{HCl}, \mathrm{pH} 7.6,150 \mathrm{mM} \mathrm{NaCl}, 0.1 \%$ Triton X-100, $0.75 \%$ Brij 96, 1 mM sodium orthovanadate, 1 $\mathrm{mM}$ sodium fluoride, $1 \mathrm{mM}$ sodium pyrophosphate, $10 \mu \mathrm{g} / \mathrm{ml}$ aprotinin, $10 \mu \mathrm{g} / \mathrm{ml}$ leupeptin, $2 \mathrm{mM}$ PMSF, and $1 \mathrm{mM}$ EDTA) for 20 minutes on ice. For immunoprecipitation to analyze protein interaction in vivo, cell lysates were precleared by incubating with normal rabbit serum and protein A/G agarose (Santa Cruz Biotechnology Inc., Santa Cruz, California, USA) on a rotator at $4^{\circ} \mathrm{C}$ overnight. The lysates were then incubated with the first protein-specific antisera (anti-14-3-3; Santa Cruz Biotechnology Inc.) for 2 hours with $50 \mu \mathrm{l}$ of protein $\mathrm{A} / \mathrm{G}$ agarose. Immune complexes were collected after each immunoprecipitation by centrifugation at $13,000 \mathrm{~g}$ for 10 minutes followed by three to five washes with lysis buffer. The immune complexes were subjected to SDS-PAGE followed by immunoblot with the second antibody, specific to ASK1 (antiASK1) (Santa Cruz Biotechnology Inc.). Chemiluminescence was detected using an ECL kit according to the instructions of the manufacturer (Amersham Life Sciences Inc., Arlington Heights, Illinois, USA). For detection of FLAG-tagged proteins (AIP1 and TRAF2), anti-FLAG M2 antibody (Sigma-Aldrich, St. Louis, Missouri, USA) was used for immunoblot. For detection of HA-tagged proteins (ASK1-WT), anti-HA antibody (Roche Diagnostics Corp., Indianapolis, Indiana, USA) was used for immunoblot.

Confocal immunofluorescence microscopy. Fixation, permeabilization, and staining of cultured HUVECs and BAECs were performed as described previously (16). For apoptotic assays, cell nuclei were stained with DAPI (2.5 $\mu \mathrm{g} / \mathrm{ml})$. Confocal immunofluorescence microscopy was performed using an Olympus confocal microscope.

$R N A$ interference constructs for Shag-AIP1. The sequences of the oligonucleotides used to create PShag-AIP1 were: AIP1-A1, 5'-ACT CCT TCA GCC TCG GCA TCA GAT GGG AGA AGC TTG TTC CGT CTG ATG CTG AGG CTG AAG GGG TCT CTT TTT T; AIP1-A2, 5'-GAT CAA AAA AGA GAC 
CCC TTC AGC CTC AGC ATC AGA CGG AAC AAG CTT CTC CCA TCT GAT GCC GAG GCT GAA GGA GTC G; AIP1-B1, 5'-ATG TGC TCC ACA CGC CGG CTG TTG TCC TGA AGC TTG AGG ATA ATA GTC GGC GTG TGG AGC ATA TCC TTT TTT T; AIP1-B2, 5'-GAT CAA AAA AAG GAT ATG CTC CAC ACG CCG ACT ATT ATC CTC AAG CTT CAG GAC AAC AGC CGG CGT GTG GAG CAC ATC G; AIP1-C1, 5'-TCC ACC TCT GAC ATC ATC AGG TCT GTC AGA AGC TTG TGA CGG ATC TGA TGA TGT CGG AGG TGG ATC GTT TTT T; and AIP-C2, 5'-GAT CAA AAA ACG ATC CAC CTC CGA CAT CAT CAG ATC CGT CAC AAG CTT CTG ACA GAC CTG ATG ATG TCA GAG GTG GAC G.

The oligonucleotides were synthesized by Integrated DNA Technology Inc. (Skotie, Illinois, USA) and were annealed/cloned into pShag vector (17). Expression of short hairpin RNA (shRNA) for AIP1 is under control of the U6 promoter.

RNase protection assay. HeLa cells were transfected with pShag or pShag-AIP1, and stable cell lines were obtained by selection in the presence of G418 (800 $\mu \mathrm{g} / \mathrm{ml}$ ). Total RNA from pooled clones was used to determined the expression of shRNA by RNase protection assay using in vitro-transcribed RNA containing AIP1 shRNA ( $400 \mathrm{bp}$ ) as a probe. Total RNA from HeLa cells was extracted with a Total RNA Isolation Kit (Ambion Inc., Austin, Texas, USA). RNase protection assays were performed using the HybSpeed RNase protection assay kit (Ambion Inc.) according to the manufacturer's directions. Each U6-hairpin cassette was cloned into NotI-EcoRV-cut pBluescript II (Promega Corp., Madison, Wisconsin, USA), linearized with $N d e I$, and radiolabeled using the MAXIscript T7/T3 in vitro transcription kit (Ambion Inc.) according to the manufacturer's instructions to generate probes for use in the RNase protection assay.
Briefly, $10 \mu \mathrm{g}$ of RNA per sample was hybridized with each probe, digested with an RNase mixture, and separated on an $8 \%$ acrylamide/8 M urea gel, followed by autoradiography as described previously (18).

\section{Results}

A novel AIP1 isolated by the yeast two-bybrid system associates with ASK1 through a conserved C2 domain. To identify ASK1 regulatory proteins, we used the C-terminal domain of ASK1 as bait in the yeast two-hybrid system. Among $2 \times 10^{6}$ transformants screened from a human cardiac library, ten clones were positive for growth on four-dropout medium (QDO) and for $\beta$-galactosidase. Sequence analysis revealed that six of the isolated cDNA's encoded the N-terminal segment (AAs 1-186) of human cDNA encoding for a novel Ras GTPase-activating protein (Ras-GAP) (GenBank accession no. AY032952) which named AIP1 (ASK1-interacting protein 1). Recently this protein has also been shown to interact with DAB2 $(14,15)$. A BLAST database search indicated that AIP1 consists of several conserved structural domains: the pleckstrin homology (PH), PKC-conserved region 2 (C2), and Ras-GAP at the N-terminal half, and at the $\mathrm{C}$-terminal half, a proline-rich sequence and a leucine-zipper motif (Figure 1a). The cDNA isolated by yeast two-hybrid screening corresponds to AIP1-PHC2 (Figure 1a). We obtained the full-length cDNA for AIP1 from HUVEC cDNA by RT-PCR (see Methods). We constructed FLAG-tagged expression plasmids for various AIP1 domains: AIP1-F (full-length, AAs 1-1,065), AIP1-C (the C-terminal half, AAs 523-1,065), AIP1-N (the N-terminal half, AAs 1-522), AIP1-PHC2 (the PH and C2 domains, AAs 1-186), and AIP1-PH (PH domain only, AAs 1-80) (Figure 1a). To determine whether AIP1 interacts with ASK1 in ECs,
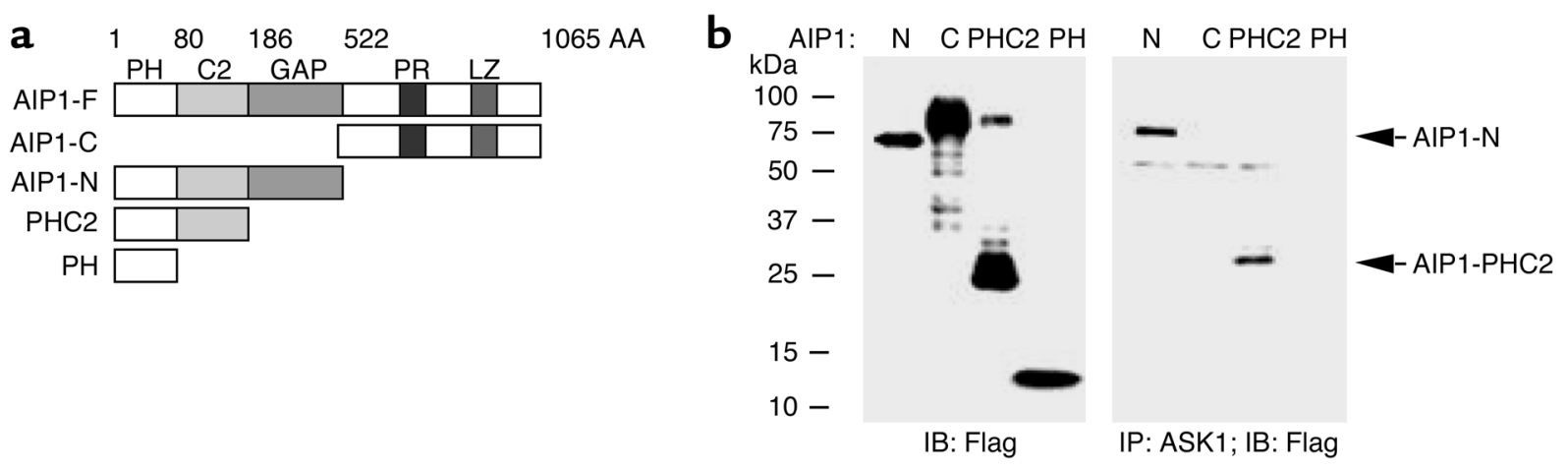

\section{Figure 1}

The C2 domain of AIP1 is critical for ASK1 binding. (a) Schematic diagram of AIP1 domains and expression constructs. The full-length AIP1 (AIP1-F) contains an N-terminal (AIP1-N) and a C-terminal half (AIP1-C). AIP1-N consists of a PH, a C2, and a GAP domain. The C-terminal half contains a proline-rich sequence (PR) and a leucine-zipper motif(LZ). AIP1-PHC2 contains the PH and C2 domains. AIP1-PH contains the $\mathrm{PH}$ domain only. AA, amino acid. (b) The C2 domain of AIP1 is critical for ASK1 binding. BAECs were transfected with FLAG-tagged AIP1-N, AIP1-C, AIP1-PHC2, or AIP1-PH, and expression of AIP1 was determined by Western blot with anti-FLAG. Interaction of AIP1 domains with endogenous ASK1 was examined by immunoprecipitation with anti-ASK1, followed by Western blot with anti-FLAG. ASK1 protein in immunoprecipitates was determined by Western blot with anti-ASK1. 


\section{a}

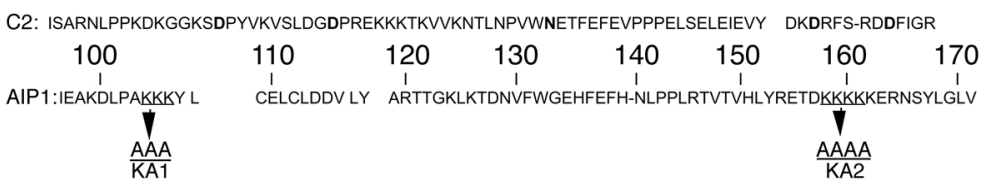

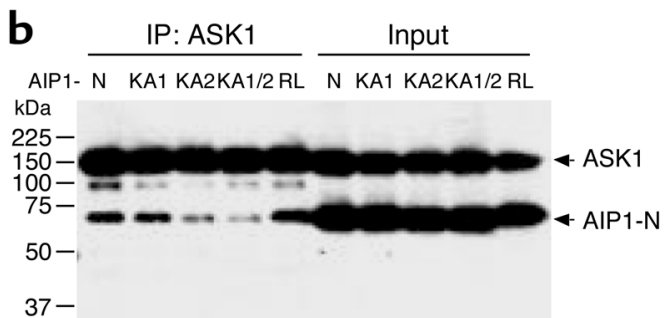

IB: Flag

Figure 2

A lysine-rich cluster within the C2 domain of AIP1 is critical for ASK1 binding. (a) Sequence alignment of C2 domains in AIP1 and a conserved C2 domain of PKC. The amino acid residues in bold are $\mathrm{Ca}^{2+}$-binding sites. The K clusters in AIP1 are underlined and are mutated to A to generate $K A 1, K A 2$, and KA1/2 (mutations at both $\mathrm{K}$ clusters). (b) Mutations at the second $\mathrm{K}$ cluster significantly reduce ASK1 binding. FLAG-tagged AIP1-N, KA1, KA2, or KA1/2 ASK1- $\Delta \mathrm{N}$ was cotransfected with ASK1 in ECs, and interaction of ASK1 with AIP1-N or mutants was examined by immunoprecipitation with anti-ASK1, followed by Western blot with anti-FLAG.

ECs were transfected with various FLAG-AIP1 constructs, and expression of AIP1 was detected by Western blot with anti-FLAG (Figure 1b). Interaction of AIP1 with endogenous ASK1 was determined by coimmunoprecipitation with anti-ASK1 followed by Western blot with anti-FLAG. Results indicated that AIP1-N and AIP1-PHC2, but not AIP1-PH or AIP1-C, interact with ASK1 (Figure 1b), suggesting that the $\mathrm{C} 2$ domain of AIP1 is critical for ASK1 binding. Further experiments showed that AIP1 interacts with ASK1- $\Delta \mathrm{N}$ but not ASK1-N or ASK1-K (not shown). These data indicate that association of AIP1 with ASK1 is through the C2 domain of AIP1 and the C-terminal domain of ASK1.

The lysine-rich cluster within the $C 2$ domain, but not GAP activity of AIP1, is critical for ASK1 binding. The C2 domain is a conserved domain present in phospholipases, PKC, and synaptotagmins, among others. It was initially identified as a $\mathrm{Ca}^{2+}$-binding motif formed by aspartic acid residues and a phosphatidyl-binding motif formed by lysine-rich clusters. However, some $\mathrm{C} 2$ domains do not contain $\mathrm{Ca}^{2+}$-binding sites but retain abilities to bind phospholipids, inositol polyphosphates, and intracellular proteins. The lysine-rich clusters within the $\mathrm{C} 2$ domain are essential for these activities (19). We compared the C2 domain in AIP1 and a typical $\mathrm{C} 2$ domain of PKC, and found that AIP1-C2 does not contain the conserved $\mathrm{Ca}_{2}{ }^{+}$-binding residues but has two lysine-rich clusters (K104-106 and K158162; Figure 2a). To examine whether the lysine-rich clusters are responsible for ASK1 association, lysine residues within the two clusters were mutated to alanine to generate three AIP1-N mutants (AIP1-KA1 with $\mathrm{K} \rightarrow \mathrm{A}$ at positions $104-106$, AIP1-KA2 with $\mathrm{K} \rightarrow \mathrm{A}$ at positions $158-161$, and $\mathrm{KA} 1 / 2$ with mutation at both clusters). We also generated a cDNA with a mutation at R289 within the GAP domain of AIP-N, (AIP1-RL), which has been shown to disrupt the GAP activity of DIP1/2, the rat homologue of AIP1 (15). ECs were transfected with FLAG-tagged AIP1-N constructs (AIP1-KA1, -KA2, -KA1/2, or -RL). Association of AIP1-KA with ASK1 was determined by coimmunoprecipitation assay with anti-ASK1 followed by
Western blot with anti-FLAG. Results showed that a mutation at R289 (AIP1-RL) had no effect on the binding of AIP1-N to ASK1 (Figure 2b), suggesting that the GAP activity of AIP1 is not crucial for ASK1 binding. However, mutations at K154-161 (AIP1-KA2 or -KA1/2), but not at K104-106 (KA1) within the C2 domain of AIP1, significantly reduced association of AIP1 with ASK1. These data suggest that K154-161 residues in the $\mathrm{C} 2$ domain of AIP1 are critical for ASK1 binding.

TNF- $\alpha$ induces association of AIP1 with ASK1 in ECs. During the coimmunoprecipitation assay, we did not observe association of the full-length AIP1 (AIP1-F) with ASK1. We therefore reasoned that AIP1-F binds to ASK1 only after being activated by extracellular stimuli. To test this hypothesis, we examined association of AIP1-F with ASK1 in response to TNF- $\alpha$, a proinflammatory cytokine inducing ASK1 activation in ECs. ECs were transfected with AIP1-F or AIP1-N and then treated with TNF- $\alpha$. The association of AIP1 with endogenous ASK1 was examined by immunoprecipitation with anti-ASK1 followed by Western blot with antiFLAG. Results showed that association of AIP1-F with ASK1 was strongly induced by TNF- $\alpha$ (Figure 3a). In contrast, AIP1-N constitutively binds to ASK1, suggesting that AIP1-C is an inhibitory domain blocking AIP1-ASK1 interaction.

To determine whether endogenous association of AIP1 and ASK1 in ECs is also TNF- $\alpha$-dependent, we generated a rabbit polyclonal antibody against the $\mathrm{N}$-terminal PH domain of AIP1 by using GST-AIP1-PH as an antigen (anti-AIP1-N; see Methods). First we determined the antibody specificity using cell lysates containing FLAG-tagged AIP1-F, AIP1-N, and AIP1-C overexpressed in 293T cells (in which endogenous AIP1 is expressed below detectable levels). Results showed that AIP1-F and AIP1-N were detected by anti-AIP1-N, but AIP1-C was not (Figure 3b). In control experiments, anti-FLAG detected AIP1-F, AIP1-N, and AIP1-C (Figure $3 \mathrm{~b})$. Previously we generated the antibody by immunizing rabbits with the C-terminal sequence corresponding to AAs 976-996 of rat DIP1/2, which is identical to 
human AIP1 (anti-AIP1-C) $(14,15)$, and this antibody recognized AIP1-F and AIP1-C (not shown). These data confirmed the specificity of anti-AIP1-N and antiAIP1-C antibodies of AIP1. We then determined expression of endogenous AIP1 in ECs. Western blot with antiAIP1 indicated that AIP1 is highly expressed in cultured HUVECs (Figure 3c) and BAECs (not shown), but not in several tumor cells, including prostate cell line LNCaP and breast cancer cell line MCF-7 (Figure 3c).

We then examined association of endogenous AIP1 and ASK1 in ECs. HUVECs were treated with TNF- $\alpha$ $(10 \mathrm{ng} / \mathrm{ml}$ for 15 minutes), and endogenous AIP1-ASK1 and ASK1-14-3-3 complexes in ECs were determined by coimmunoprecipitation with anti-ASK1, followed by Western blot with anti-AIP1 or 14-3-3. TNF- $\alpha$ treatment did not significantly alter expression of ASK1, AIP1, or 14-3-3 in ECs (Figure 3c). However, association of AIP1 with ASK1 was strongly increased by TNF- $\alpha$ treatment, suggesting that association of AIP1 with ASK1 is TNF- $\alpha$-inducible. In contrast, TNF- $\alpha$ reduced association of 14-3-3 with ASK1, consistent with the model that TNF- $\alpha$ activates ASK1 in part by dissociating ASK1 from 14-3-3 (Figure 3d). Similar results were obtained in BAECs (not shown).

TRAF2 is a critical adapter protein mediating TNF- $\alpha-$ induced ASK1 activation $(9,12)$, and overexpression of TRAF2 in ECs activates the ASK1-JNK pathway $(6,7)$. We examined whether overexpression of TRAF2 induces AIP1 association with ASK1. BAECs were transfected with vector control or FLAG-tagged TRAF2, and endogenous AIP1-ASK1 complex was determined. Results show that formation of AIP1-ASK1 complex was significantly increased by TRAF2 (Figure 3e). We also examined the effect of AIP1 on the TRAF2-ASK1 complex. BAECs were transfected with vector control or AIP1-N, followed by TNF- $\alpha$ treatment $(10 \mathrm{ng} / \mathrm{ml})$, and endogenous TRAF2-ASK1 complex was determined by immunoprecipitation with anti-TRAF2, followed by Western blot with anti-ASK1. As expected, formation of TRAF2-ASK1 complex is induced by TNF- $\alpha$ in ECs. AIP1-N expression did not increase the basal or TNF- $\alpha-$ induced TRAF2-ASK1 interaction (Figure 3f).

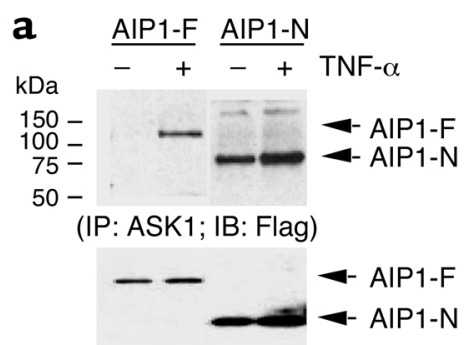

(IB: Flag)

(IB: ASK1)
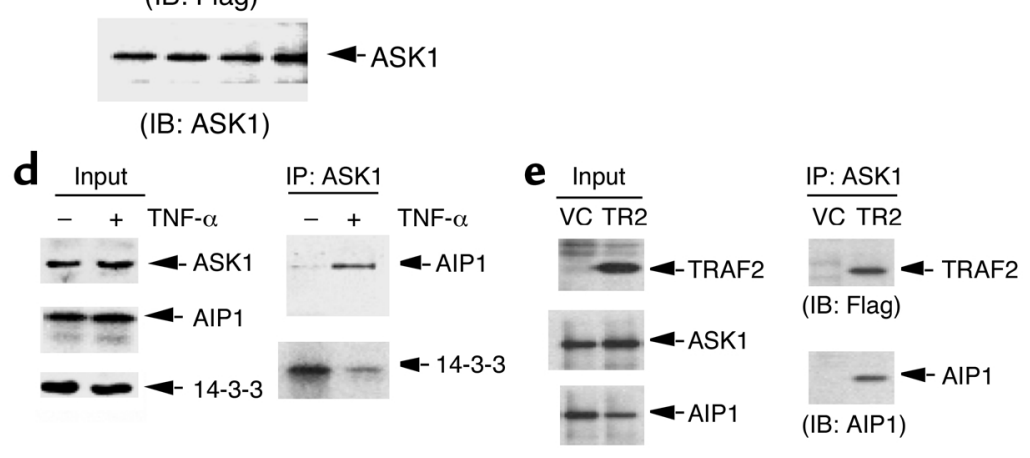

C Ctrl ECMCF-7 LNCaP

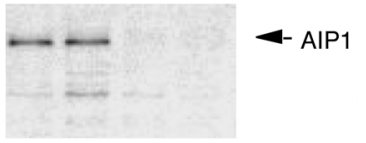

(IB: AIP1)

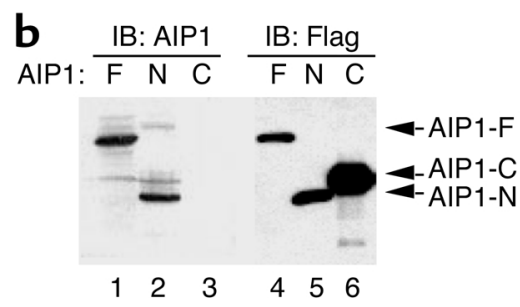

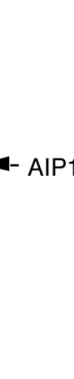

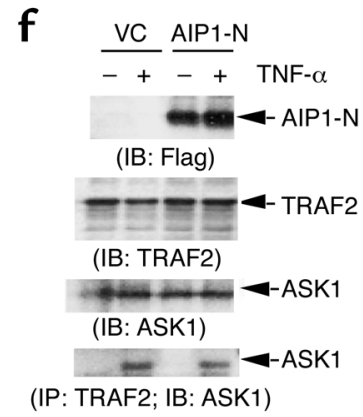

Figure 3

TNF- $\alpha$ induces association of AIP1 with ASK1 in ECs. (a) BAECs were transfected with AIP1-F or AIP1-N followed by treatment with TNF- $\alpha$ $(10 \mathrm{ng} / \mathrm{ml}$ for 15 minutes). Cell lysates were immunoprecipitated with anti-ASK1, and ASK1 in the immunoprecipitate was determined by Western blot with anti-AIP1. (b) Specificity of AIP1 antibody. A polyclonal antibody against AIP1 was produced by Cocalico Biologicals Inc. by immunizing rabbits with GST-AIP1-PH. 293T cell lysates expressing FLAG-tagged AIP1-F, -N, and -C were used to determine the specificity of anti-AIP1 by Western blot (lanes 1-3). Anti-FLAG was used as a control (lanes 4-6). (c) AIP1 is highly expressed in cultured ECs. AIP1 expression in cell lysates from HUVECs, breast cancer MCF-7 cells, or prostate cancer cell line LNCaP (20 $\mu$ g of total protein from each sample) was measured by Western blot with anti-AIP1. (d) TNF- $\alpha$ induces association of AIP1 with ASK1, whereas it dissociates 14-3-3 from ASK1. HUVECs were either untreated or treated with TNF- $\alpha$ (10 ng/ml for 15 minutes). Cell lysates were immunoprecipitated with anti-ASK1 followed by Western blot with anti-AIP1 or anti-14-3-3. (e) TRAF2 induces association of AIP1 with ASK1. BAECs were transfected with vector control (VC) or FLAG-tagged TRAF2 (TR2). Association of TRAF2 or AIP1 with ASK1 was determined by immunoprecipitation with anti-ASK1, followed by Western blot with anti-FLAG or anti-AIP1. (f) AIP1 has no effect on TRAF2-ASK1 complex. BAECs were transfected with vector control or AIP1-N, followed by TNF- $\alpha$ treatment $(10 \mathrm{ng} / \mathrm{ml})$. Endogenous TRAF2-ASK1 complex was determined by immunoprecipitation with anti-TRAF2 followed by Western blot with anti-ASK1. IP, immunoprecipitate; IB immunoblot. 


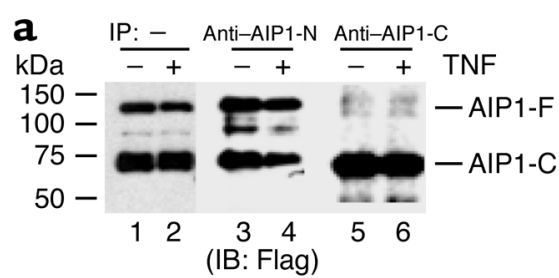

b

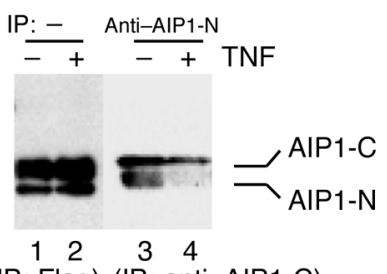

(IB: Flag) (IB: anti-AIP1-C)

\section{Figure 4}

TNF- $\alpha$ disrupts intramolecular interaction between the $\mathrm{N}$-terminus and $\mathrm{C}$-terminus of AIP1. (a) AIP1 folds in a closed form. BAECs were transfected with AIP1-F and AIP1-C, followed by treatment with $10 \mathrm{ng} / \mathrm{ml} \mathrm{TNF-} \alpha$ for 15 minutes (+) or no treatment $(-)$. Cell lysates were immunoprecipitated with either anti-AIP1-N or anti-AIP1-C of AIP1, followed by Western blot with anti-FLAG. (b) TNF- $\alpha$ disrupts interaction of AIP1-C and AIP1-N. ECs were transfected with AIP1-C and AIP1-N followed by TNF- $\alpha$ treatment as in a (+). Expression of AIP1-C and AIP1-N was detected by Western blot with anti-FLAG. Cell lysates were immunoprecipitated with anti-AIP1-N, followed by Western blot with anti-AIP1-C (lanes 3-4).

TNF- $\alpha$ unfolds the intramolecular interaction between the $N$-terminus and C-terminus of AIP1. TNF- $\alpha$-induced binding of AIP1 to ASK1 suggested to us that AIP1 is inactive due to intramolecular interactions and that TNF- $\alpha$ causes AIP1 to unfold and become active. This was first supported by our finding that anti-AIP1-N antibody (recognizing the $\mathrm{N}$-terminal $\mathrm{PH}$ domain), but not anti-AIP1-C antibody (recognizing the C-terminal domain), could efficiently immunoprecipitate AIP1-F (Figure 4a, lane 3 vs. lane 5). Anti-AIP1-N also immunoprecipitated AIP1-C in the presence of AIP1-F, suggesting that AIP1-F and AIP1-C form a complex (Figure 4a, lane 3). TNF- $\alpha$ treatment $(10 \mathrm{ng} / \mathrm{ml}$ for 15 minutes) significantly reduced AIP1-F-AIP1-C complex formation (Figure 4a, lane 4 vs. lane 3 ). To determine whether AIP1-C binds to the $\mathrm{N}$-terminal domain or the C-terminal domain (dimerization) of AIP1-F, we examined interaction of AIP1-N and AIP-C. ECs were cotransfected with AIP1-C and AIP1-N followed by TNF- $\alpha$ treatment $(10 \mathrm{ng} / \mathrm{ml}$ for 15 minutes). Association of AIP1-C and AIP1-N was determined by immunoprecipitation with anti-AIP1-N followed by Western blot with anti-AIP1-C. Results showed that AIP1-N and AIP1-C form a complex and that TNF- $\alpha$ significantly reduced the amount of complex (Figure $4 b)$. These data suggest that TNF- $\alpha$ promotes a disruption of the intramolecular interaction between the $\mathrm{N}$-terminus and C-terminus of AIP1.

AIP1 disrupts the ASK1-14-3-3 complex. The data indicating that AIP1 and 14-3-3 associate with ASK1 in a reciprocal manner prompted us to reason that AIP1 competes with 14-3-3 for ASK1 binding in response to TNF- $\alpha$. To test this hypothesis, ECs were transfected with expression plasmids for FLAG-tagged 14-3-3 (1 $\mu \mathrm{g})$ and with increasing amounts of FLAG-AIP1-N $(0-1 \mu \mathrm{g})$. ASK1-associated AIP1 and 14-3-3 were determined by immunoprecipitation with anti-ASK1, followed by Western blot with anti-FLAG. Expression of AIP1-N had no effect on the expression level of ASK1 and 14-3-3 (Figure 5a). However, binding of 14-3-3 to ASK1 was dramatically reduced, concomitant with an increase of ASK1-AIP1-N complex formation (Figure $5 \mathrm{a})$. To exclude the possibility that AIP1-N competes with ASK1 for 14-3-3 binding, we examined interaction of AIP1-N with 14-3-3 in a GST-14-3-3 pulldown assay. Results showed that 14-3-3 did not bind to AIP1-N (Figure 5b). In control experiments, ASK1 was shown to bind to GST-14-3-3 as described previously (6). These data strongly suggest that AIP1 can dissociate 14-3-3 from ASK1 in vivo.

\section{Figure 5}

Overexpression of AIP1 in ECs releases 14-3-3 from ASK1. (a) AIP1 dissociates 14-3-3 from ASK1. BAECs were transfected with expression plasmids for FLAGtagged 14-3-3 $(1 \mu \mathrm{g})$ with various amounts of FLAGAIP1-N DNA $(0,0.1,0.25,0.5,0.75$, and $1 \mu \mathrm{g})$. Association of AIP1 and 14-3-3 with endogenous ASK1 was determined by immunoprecipitation with anti-ASK1 followed by Western blot with anti-FLAG. ASK1 in the immunoprecipitates was determined by Western blot with anti-ASK1. (b) AIP1-N does not bind to 14-3-3. Cell lysate expressing AIP1-N was used in a GST-14-3-3 pulldown assay. Bound AIP1-N was detected by Western blot with anti-FLAG. ASK1 was used as a control.

\section{a}$$
\text { 14-3-3 }
$$

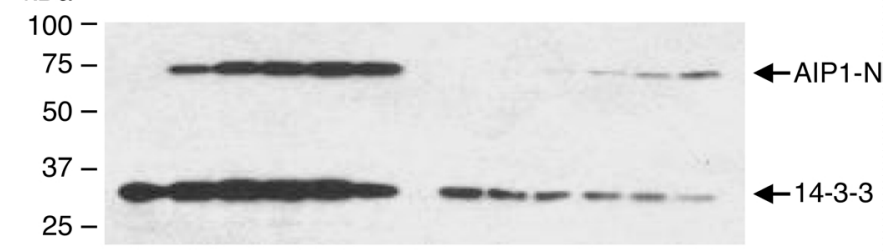

(IB: Flag)

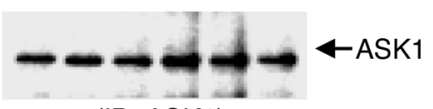

(IB: ASK1)

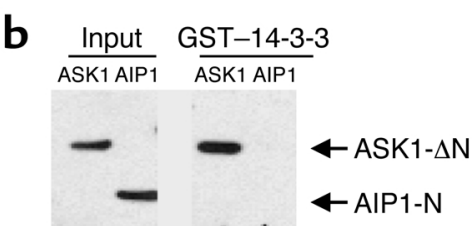

(IB: Flag) 
AIP1 binds preferentially to dephosphorylated ASK1 at Ser967. Previously we have shown that 14-3-3 associates with ASK1 via pSer-967 and that TNF- $\alpha$ activates ASK1 in part by dephosphorylating ASK1 at Ser-967, leading to release of 14-3-3. To determine how AIP1 mediates the TNF- $\alpha$-induced release of 14-3-3 from ASK1, we performed a series of experiments to examine the role of pSer-967 in AIP binding. First, we examined association of AIP1 with ASK1-S967A, a mutant ASK1 defective in 14-3-3 binding as shown previously (6). AIP1-N was cotransfected with ASK1 or ASK1-S967A in BAECs, and association of AIP1-N with ASK1 was determined by immunoprecipitation with anti-ASK1, followed by Western blot with anti-FLAG. Results showed that AIP1 strongly associates with both ASK1-WT and ASK1-S967A, with ASK1-S967A being the stronger (Figure 6a). These data suggest that pSer-967 of ASK1 is not critical for AIP1 binding.

Overexpressed ASK1-WT may exist in both phosphorylated and dephosphorylated forms at the Ser-967 site. To distinguish which form of ASK1 interacts with AIP1, we treated ASK1 with alkaline phosphatase (PPase). Treatment of cell lysates containing ASK1-WT and AIP1-N with PPase (10 U PPase/40 $\mu \mathrm{g}$ cell lysate at room temperature for 1 hour) significantly increased formation of AIP1-ASK1 complex, as measured in the immunoprecipitation assay (Figure $6 \mathrm{~b}$ ). In contrast, PPase treatment significantly reduced binding of ASK1 to 14-3-3 in a GST-14-3-3 pull-down assay (Figure 6b). These data suggest that AIP1 preferentially binds to dephosphorylated ASK1.

To further determine the role of ASK1's pSer-967 in AIP1 binding, we performed a peptide competition assay using 15 -mer peptides flanking the pSer-967 of ASK1 (AEYLRSIS ${ }^{967}$ LPVPVLV) in two forms: nonphosphorylated (ASK1-S) and phosphorylated peptides (ASK1-pS). Peptide specificity was first determined in a GST-14-3-3 pull-down assay for ASK1 binding in the presence of $0.3 \mathrm{mM}$ of either ASK1-S or ASK1-pS. As expected, ASK1-pS, but not ASK1-S, blocked binding of ASK1 to GST-14-3-3, confirming that 14-3-3 binds to ASK1 via pSer-967 (Figure 6c). We then examined the effects of these peptides on AIP1-ASK1 complex formation. AIP1 and ASK1 were coexpressed in ECs, and AIP1-ASK1 complexes were determined by immunoprecipitation with anti-ASK1 in the presence of ASK1-pS or ASK1-S. Associated AIP1 was determined by Western blot with anti-FLAG. In contrast to the results from the 14-3-3 pull-down assay, ASK1-S, but not ASK1-pS, disrupted the AIP1-ASK1 complex (Figure 6d). These

Figure 6 data suggest that AIP1 binds to a sequence surrounding the 14-3-3 binding site with preference for the dephosphorylated form of ASK1 at Ser-967.

AIP1 enhances TNF- $\alpha$-induced ASK1-JNK activation. To determine whether AIP1 facilitates dissociation of 14-3-3 from ASK1, leading to enhanced biological activities of ASK1, we examined effects of AIP1 on ASK1 kinase activity, ASK1-induced JNK activation, and EC apoptosis. We first determined the effects of AIP1 on TNF- $\alpha-$ induced ASK1-JNK activation. BAECs were transfected with AIP1 constructs (AIP1-F, AIP1-N, and AIP1-C) in the absence or presence of TNF- $\alpha$ treatment $(10 \mathrm{ng} / \mathrm{ml}$ for 15 minutes). Expression of AIP1 was determined by Western blot with anti-FLAG (Figure 7a). ASK1 protein was immunoprecipitated by anti-ASK1, and effects of AIP1 on ASK1 activity in the immunocomplex were determined by an in vitro kinase assay using GST-MKK4 (JNKK1) as a substrate. Results show that AIP1-N significantly increased ASK1 activity and AIP1-F did so to a lesser extent, but AIP1-C had no such effect (Figure 7b, top panel). This is consistent with the abilities of AIP1's to associate with ASK1 in the immunocomplex (see Figure 1). ASK1-induced JNK activation was measured by a
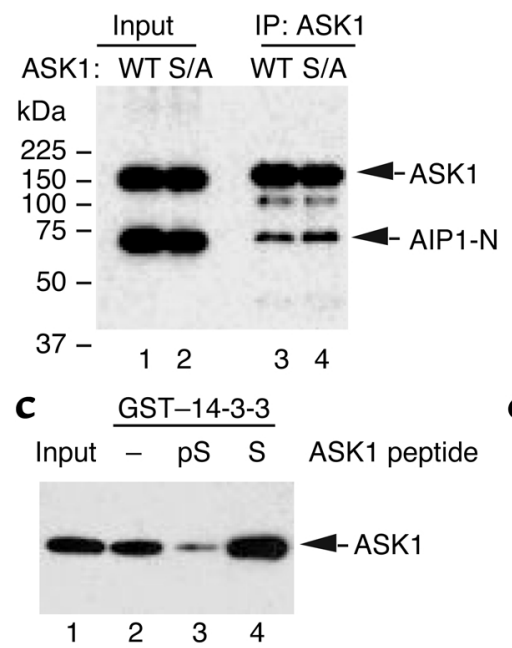

b

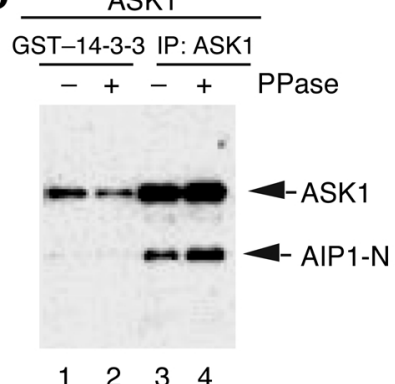

d

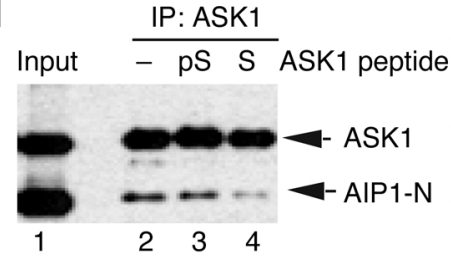

AIP1 binds preferentially to dephosphorylated ASK1 at Ser-967. (a) The 14-3-3 binding site ( $p S e r-967)$ in ASK1 is not critical for AIP1 association. AIP1 was cotransfected with vector control, ASK1, or ASK1-S967A, a mutant defective in 14-3-3 binding. Interaction of AIP1 and ASK1 was examined by immunoprecipitation with anti-ASK1 followed by Western blot with anti-FLAG. S/A, ASK1-S967A. (b) Phosphatase treatment increases AIP1-ASK1 complex. Cell lysates containing ASK1 and AIP1-N were incubated with alkaline phosphatase ( $10 \mathrm{U}$ PPase $/ 40 \mu \mathrm{g}$ cell lysate) at room temperature for 1 hour. Treated lysates were used IP by anti-ASK1, followed by Western blot with anti-FLAG. A GST-14-3-3 pull-down assay was used as a control for phosphatase treatment. Bound ASK1 was detected by Western blot with anti-FLAG. (c) Peptide specificity. ASK1 was used in a GST-14-3-3 pull-down assay in the presence of peptide ASK1-S or ASK1-pS (0.3 mM). Bound ASK1 was detected by Western blot with anti-FLAG. (d) Peptide competition assay for AIP1-ASK1 interaction. Cell lysates containing ASK1 and AIP1-N were used for immunoprecipitation assay as described in $\mathbf{a}$ in the presence of peptide ASK1-S or ASK1-pS (0.3 $\mathrm{mM}$ ). Interaction of AIP1 and ASK1 was examined by immunoprecipitation with anti-ASK1, followed by Western blot with anti-FLAG. 

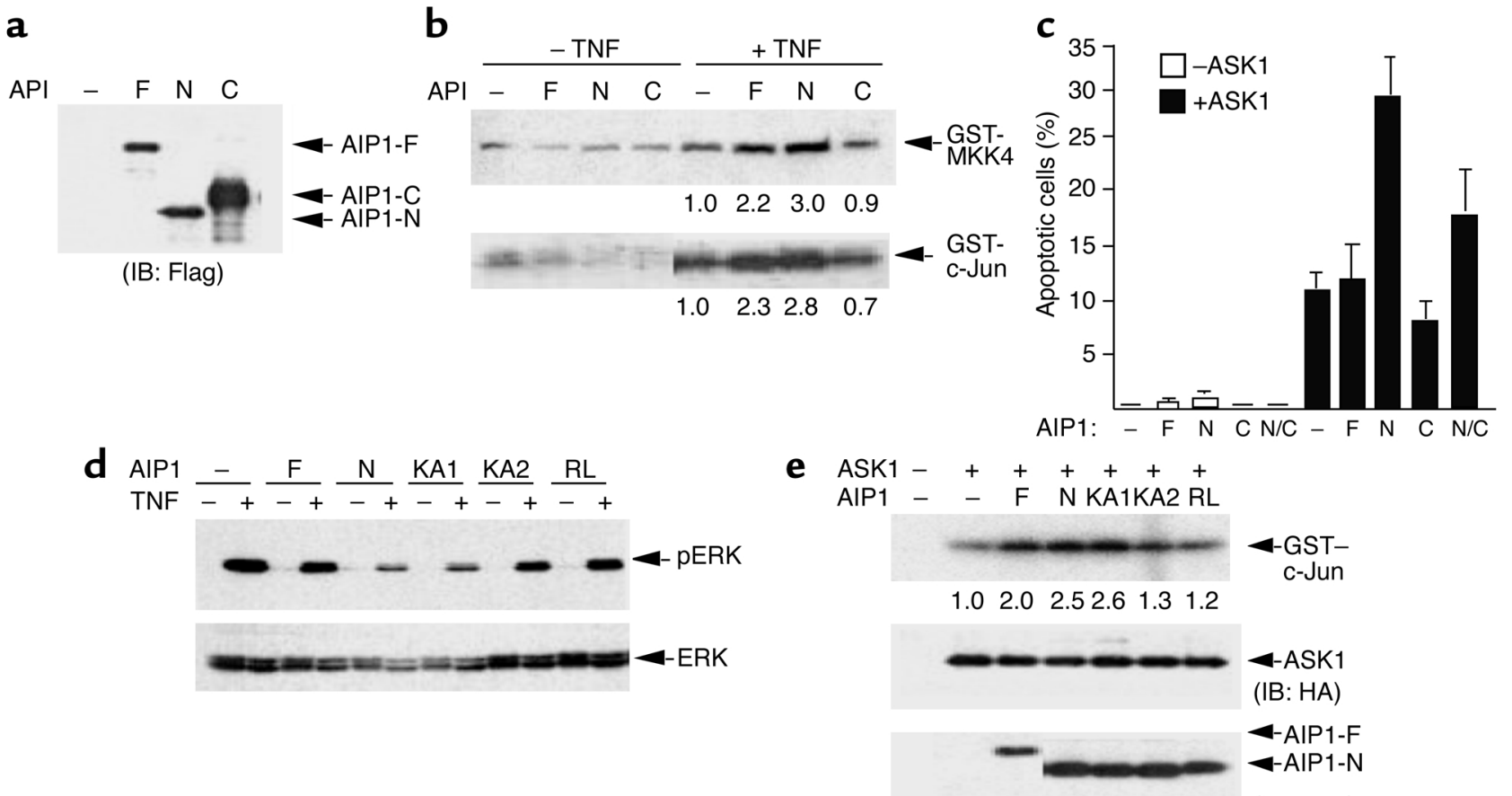

(IB: Flag)

\begin{abstract}
Figure 7
AIP1 enhances TNF- $\alpha$-induced ASK1 activity. ( $\mathbf{a}$ and $\mathbf{b})$ AIP1 enhances TNF- $\alpha$-induced ASK1 and JNK activation. BAECs were transfected with vector control (-) or AIP1 constructs (AIP1-F, -N, or -C). Cell lysates were used to determine protein expression by Western blot with anti-FLAG (a) ASK1 and JNK activation were measured by an in vitro kinase assay (b). The relative ASK1 and JNK activities (setting TNF- $\alpha$-treated vector control as 1.0) are indicated below each lane. (c) AIP1 enhances ASK1-induced EC apoptosis. BAECs were transfected with vector control (-) or AIP1 constructs (AIP1-F, AIP1-N, and AIP1-C) in the absence or presence of ASK1. Forty-eight hours after transfection, cells were stained with DAPI, and apoptotic cells with nucleus fragmentation were counted under a fluorescence microscope. The apoptotic rate is shown. Data presented are mean of three independent experiments. N/C, AIP1-N + AIP1-C. (d) AIP1 inhibits TNF- $\alpha$-induced ERK activation. ECs were transfected with AIP1 constructs followed by TNF- $\alpha$ stimulation $(10 \mathrm{ng} / \mathrm{ml}$ for 15 minutes). ERK activation was determined by Western blot with phospho-ERK antibody. Total ERK was determined by Western blot with anti-ERK. Similar results were obtained from two additional independent experiments. (e) ASK1 binding and the GAP activity of AIP1 are required for AIP1-enhanced ASK1 activation. AIP1 constructs were transfected into BAECs in the presence of HA-tagged ASK1. Protein expression was determined by Western blot with anti-HA (for ASK1) or antiFLAG (for AIP1). ASK1-induced JNK activity was determined as described in b. Data shown are representative of three similar experiments.
\end{abstract}

an in vitro kinase assay using GST-c-Jun as a substrate. Results showed that expression of AIP1 alone did not significantly activate JNK (Figure 7b, lanes 1-4). However, AIP1-F and AIP1-N (but not AIP1-C) significantly enhanced TNF- $\alpha$-induced JNK activation (Figure $7 b$ ). AIP1-N showed greater ability than AIP1-WT to enhance ASK1 activity, consistent with its binding to ASK1 (Figure 7b). Finally, we determined effects of AIP1 on ASK1induced EC apoptosis. BAECs were transfected with AIP1 constructs (AIP1-F, AIP1-N, and AIP1-C) in the absence or presence of ASK1. Forty-eight hours after transfection, ECs were stained with DAPI to detect apoptotic cells, which show nuclei fragmentation under a fluorescence microscope. Results show that AIP1-N significantly increased ASK1-induced EC apoptosis (by 2.8-fold) (Figure 7c). Coexpression of AIP1-C suppressed AIP1-N activity, consistent with the fact that AIP1-C could form a complex with AIP1-N (see Figure 4).

We further examined the role of the ASK1-binding motif and the GAP activity in AIP1-enhanced ASK1 activity (JNK activation). We chose AIP1-KA2 (defective in ASK1 binding) and AIP1-RL for our study. We previously showed that AIP1 homologue (rat DIP1/2) has GAP activity toward Ras, inhibiting EGF-induced ERK activation; a point mutation (R220L, equivalent to human AIP1 R289L) at the GAP domain diminished the GAP activity (15). We first examined the effect of AIP1 GAP on TNF- $\alpha$-induced ERK activation in ECs. BAECs were transfected with AIP1 constructs (AIP1-F, -N, -KA1, -KA2, and -RL) using Lipofectamine 2000, which usually yielded $90 \%$ transfection efficiency. This high transfection efficiency allowed us to examine the effects of transgenes on endogenous protein activities, as we described previously $(7,20)$. Transfected ECs were serum starved and then stimulated with TNF- $\alpha$ (10 $\mathrm{ng} / \mathrm{ml}$ for 15 minutes). ERK activation was determined by Western blot with phospho-ERK antibody. Results showed that TNF- $\alpha$ significantly induced ERK activation (Figure 7c), as described previously (21). AIP1-N is much stronger than AIP1-F in inhibiting TNF- $\alpha-$ induced ERK activation, suggesting that AIP1-N is a constitutively active form (Figure 7d). Mutation at 
lysine cluster 1 within the $\mathrm{C} 2$ domain (AIP1-KA1) had no effect on AIP1-N activity. However, AIP1-N mutants defective in either ASK1 binding (AIP1-KA2) or GAP activity (AIP1-RL) had significantly reduced ability to inhibit TNF- $\alpha$-induced ERK activation (Figure $7 d$ ). These data suggest that AIP1 functions as a GAP to inhibit TNF- $\alpha$-induced ERK in ECs and that the ASK1-binding activity and the GAP activity of AIP1 are critical for this inhibitory activity.

We then examined the effects of these AIP1 mutants on ASK1 activity. ECs were transfected with AIP1 constructs (AIP1-F, -N, -KA1, -KA2, and -RL) in the presence of HA-tagged ASK1. We used HA-ASK1 to separate ASK1 from AIP1-F because the two run very closely. ASK1-induced JNK activation was measured by in vitro kinase assay as described. Results showed that mutants defective in either ASK1 binding (AIP-KA2) or in GAP activity (AIP1-RL) failed to enhance ASK1 activity (Figure 7e), suggesting that both the association of AIP1 with ASK1 and the GAP activity of AIP1 are required for AIP1-induced ASK1 activation.

AIP1 knockdown suppresses TNF- $\alpha$-induced ASK1-JNK activation. To determine the physiological role of AIP1 in ASK1 activation, we further examined the effect of AIP1 in regulation of ASK1 activity in AIP1-knockdown cells. First we used an RNA interference approach to downregulate the endogenous AIP1 level. Three pairs of oligonucleotides for the shRNA of AIP1 were selected based on the method described (ShagAIP1-A, -9 to +19 overlapping the ATG site; ShagAIP1-B, +393 to +421 from the ATG site in the C2 domain; and ShagAIP1-C,
+804 to +833 in the GAP domain) (17). The doublestrand oligonucleotides were synthesized and cloned into pShag vector to generate pShag-AIP1-A and pShag-AIP1-B, in which shRNA of AIP1 is driven by a U6 promoter (17). Preliminary data show that ShagAIP1-B and Shag-AIP1-C (with Shag-AIP1-B having the stronger effect), but not Shag-AIP1-A, inhibit transfected AIP1 expression by transient transfection in BAECs (not shown). We chose Shag-AIP1-A (negative) and Shag-AIP1-B (positive) for further studies on endogenous AIP1. Because the bovine AIP1 sequence is not known and AIP1 constructs are produced from human cDNA, we cannot perform RNAi in bovine ECs (BAECs with highly efficient transfection). Human ECs (HUVECs) are not used because of their low transfection efficiency. In addition, BAECs and HUVECs cannot be used to generate stable cell lines because they are primary culture cells. We found that the human cervical carcinoma HeLa cell line and HUVECs have a similar level of expression of AIP1 and ASK1 protein (compare Figure $3 e$ and Figure $8 b$ ). Thus we chose HeLa cells to generate stable cell lines for expression of AIP1 shRNA.

Expression of AIP1 shRNA in HeLa cells (pooled clones) was determined by RNase protection assay using in vitro-transcribed RNA containing AIP1 shRNA as a probe ( $400 \mathrm{bp})$. The protected small interference RNA (SiRNA) to AIP1 ( 29 nucleotides) was detected by PAGE (Figure 8a), indicating that the transcribed shRNA was processed to SiRNA (17). We then examined the effects of AIP1 shRNA on AIP1 expression in these clones by Western blot with anti-AIP1.
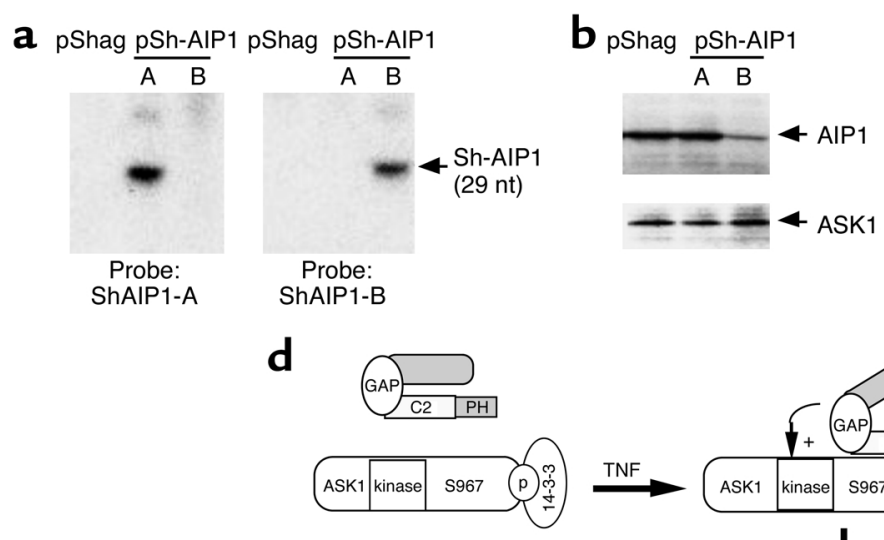
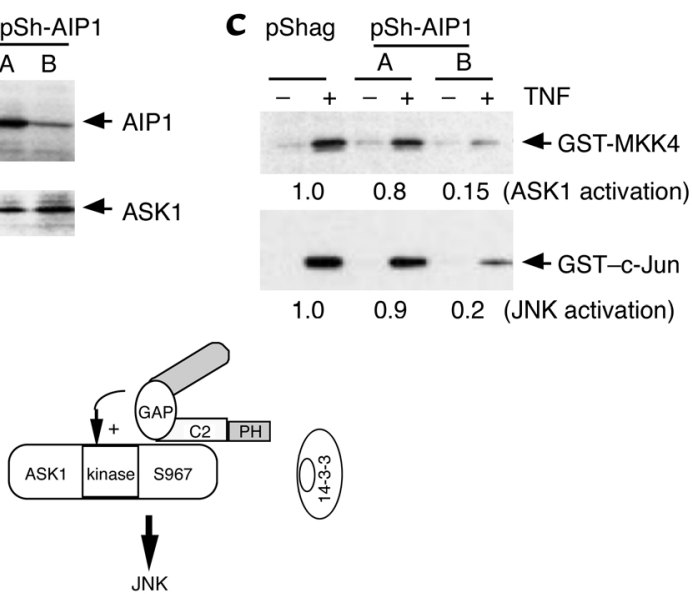

\begin{abstract}
Figure 8
Physiological role of AIP1 in TNF- $\alpha$-induced ASK1-JNK activation. (a) RNase protection assay for AIP1 shRNA expression. The protected SiRNA AIP1 probe ( 29 nucleotides) was detected by PAGE and indicated. RNA from pShag was used as a control. (b) Expression of AIP1 shRNA knockdown AIP1 protein expression. Total cell lysates from stably transfected HeLa clones (pShag, pShag-AIP1-A, or pShag-AIP-B) were used to determine AIP1 expression by Western blot with anti-AIP1. ASK1 expression was used as a control. (c) TNF- $\alpha$-induced ASK1 and JNK activation is blunted in pShag-AIP1 cells. Stable pooled HeLa cells transfected with pShag or pShag-AIP1 were untreated or treated with TNF- $\alpha$ (10 ng/ml for 15 minutes). ASK1 and JNK activities were determined by in vitro kinase assay as described. Relative ASK1 and JNK activities are shown, with activity of TNF- $\alpha$-treated $p S h a g$-transfected cells used as 1.0. Similar results were obtained from two additional experiments. (d) Proposed model for AIP1 function in TNF- $\alpha$-induced ASK1-JNK signaling. AIP1 folds in a closed form through an intramolecular interaction between the N-terminus and C-terminus. Proinflammatory mediators (e.g., TNF- $\alpha$ ) disrupt the intramolecular interaction of AIP1. TNF- $\alpha$ also induces dephosphorylation of ASK1 at Ser-967 by unidentified phosphatase(s), leading to recruitment of AIP1 and release of 14-3-3. Recruited AIP1 enhances TNF- $\alpha$-induced ASK1-JNK signaling in which both ASK1 binding and the GAP activity of AIP1 are required.
\end{abstract}


The expression of AIP1 was significantly (80\%) blocked by pShagAIP1-B but not by pShagAIP1-A (Figure 8b). In contrast, ASK1 was not altered by expression of AIP1 shRNA. Five individual single clones derived from the pool of pShagAIP1-B showed similar inhibition of AIP1 expression (not shown). These data suggest that AIP1 shRNA from pShagAIP1 efficiently knocked down endogenous AIP1 expression.

We next examined the effects of AIP1 knockdown on TNF- $\alpha$-induced ASK1-JNK activation. Stable HeLa cells were untreated or treated with TNF- $\alpha$ (10 $\mathrm{ng} / \mathrm{ml}$ for 15 minutes), and ASK1-JNK activation was determined by the in vitro kinase assay as described. Results show that ASK1 and JNK activation by TNF- $\alpha$ were significantly blunted (by five- to sevenfold) in pShagAIP1-B cells compared with that in pShag or pShagAIP1-A cells (Figure 8c). Similar data were obtained for a TNF- $\alpha /$ ASK-induced JNK-dependent reporter gene (not shown), suggesting that knockdown of AIP1 reduced ASK1-JNK signaling.

\section{Discussion}

In this study we show that AIP1, a newly described Ras-GAP protein, facilitates TNF- $\alpha$-induced dissociation of ASK1 from its inhibitor 14-3-3, leading to enhanced ASK1 activation. Association of AIP1 with ASK1 and the GAP activity of AIP1 are required for AIP1-enhanced ASK1 activity. While 14-3-3 binds to phosphorylated ASK1 (pSer-967 ASK1) in resting ECs, AIP1 binds to the dephosphorylated active form of ASK1. Moreover, TNF- $\alpha$-induced ASK1-JNK activation is significantly blunted in cells where AIP1 is knocked down by RNA interference. These data suggest that AIP1 mediates TNF- $\alpha$-induced ASK1 activation by dissociating inhibitor 14-3-3 from ASK1, a novel mechanism by which TNF- $\alpha$ activates ASK1 (Figure 8d). Based on these results, we conclude that AIP1 is a novel regulator of ASK1.

14-3-3 binds to ASK1 in resting ECs, indicating that ASK1 is basally phosphorylated at Ser-967 and forms a preexisting complex with 14-3-3. TNF- $\alpha$ activates ASK1 in part by dissociating ASK1 from 14-3-3. We have previously proposed that TNF- $\alpha$ activates a phosphatase that dephosphorylates ASK1 at Ser-967 and releases ASK1 from 14-3-3 inhibitor. Phosphatases such as cdc25A and PP5 have been implicated in regulating ASK1 activation in a negative fashion $(22,23)$. The cdc25A phosphatase binds to ASK1 at a site adjacent to the kinase domain and inhibits ASK1 activity in a phosphatase activity-independent manner (19). PP5 has been shown to associate with ASK1 in response to hydrogen peroxide and to dephosphorylate ASK1 at Thr-845 to inhibit ASK1 activation as a feedback mechanism (23). The phosphatase(s) that targets ASK1 Ser967 needs to be identified. It is plausible that AIP1 recruits the phosphatase to ASK1 to facilitate 14-3-3 release. Identification of this phosphatase will help us to further understand the mechanism for AIP1-mediated 14-3-3 release from ASK1.
Recent studies have demonstrated that ASK1 activation involves several steps, including release of inhibitors (such as Trx, glutaredoxin, glutathione S-transferase- $\mu$, heat shock proteins, and 14-3-3) $(6,10,13$, 24-26), TRAF-dependent homodimerization/polymerization (11, 12), autophosphorylation at Thr-845 (27), and scaffold protein-mediated association of ASK1 with downstream MKK and JNK (28-30). Our data show that AIP1 overexpression alone does not activate the endogenous ASK1-JNK signaling pathway in ECs. It seems that release of 14-3-3 by AIP 1 is necessary but not sufficient for ASK1 activation. Release of other inhibitors such as $\operatorname{Trx}$ is also required. It is conceivable that TNF- $\alpha$ induces release of Trx and 14-3-3 in a coordinated fashion, leading to ASK1-JNK activation.

AIP1 mutants defective in ASK1 binding or GAP activity failed to increase ASK1 activity, suggesting that both association of AIP1 with ASK1 and GAP activity of AIP1 are required for AIP1-enhanced ASK1 activity. The GAP activity of Ras-GAP is known to be critical for regulation of Ras-Raf-ERK activation. The requirement for AIP1 GAP activity in ASK1-JNK activation is supported by the observation that ERK exerts an inhibitory effect on ASK1-JNK signaling. Crosstalk among MAPKs has been well documented: one MAPK (such as ERK) may inhibit or oppose the activation of another MAPK (such as JNK). Many stimuli reciprocally regulate ERK and JNK activation. We have previously shown that atheroprotective laminar flow inhibits TNF- $\alpha$-induced ASK1JNK activation, and that laminar flow-induced MEKERK activation is required for inhibition of JNK $(6,21)$. Conversely, our data support the notion that ERK inhibition by AIP1 (via GAP activity) may be required for AIP1-enhanced ASK1-JNK activation (Figure 8). Our study has established a link between Ras-GAP and the stress-activated ASK1-JNK signaling pathway. It remains to be determined whether recruitment of AIP1 to ASK1 activates the GAP activity of AIP1.

Both TNF- $\alpha$ and TRAF2 overexpression enhance association of AIP1 with ASK1. In contrast, AIP1 overexpression has no effect on TNF- $\alpha$-induced formation of TRAF2-ASK1 complex, suggesting that AIP1 is downstream of TRAF2 in ASK1 activation. The mechanism by which TNF- $\alpha$ induces unfolding of AIP1 is not clear. The role of TRAF2 in TNF-induced AIP1 unfolding is currently under investigation.

In conclusion, our data show that AIP1 facilitates dissociation of 14-3-3 from ASK1, leading to enhanced ASK1-JNK activation, and suggest that AIP1 is a novel regulator in TNF- $\alpha$-induced ASK1 activation.

\section{Acknowledgments}

We thank Gregory J. Hannon (Cold Spring Harbor Laboratory) for the pShag vector. We thank Jeff W. Streb and Chad Kitchen for technical assistance in RNA interference design and RNA protection assay. We thank Bradford C. Berk for discussion and critical reading of the manuscript. This work was supported by NIH grant 1R01 HL-65978-01 (to W. Min). 
1. Davis, R.J. 2000. Signal transduction by the JNK group of MAP kinases. Cell. 103:239-252.

2. Ichijo, H., et al. 1997. Induction of apoptosis by ASK1, a mammalian MAPKKK that activates SAPK/JNK and $\mathrm{p} 38$ signaling pathways. Science. 275:90-94.

3. Chang, H.Y., Nishitoh, H., Yang, X., Ichijo, H., and Baltimore, D. 1998. Activation of apoptosis signal-regulating kinase 1 (ASK1) by the adapter protein Daxx. Science. 281:1860-1863.

4. Takeda, K., et al. 2000. Apoptosis signal-regulating kinase 1 (ASK1) induces neuronal differentiation and survival of PC12 cells. J. Biol. Chem. 275:9805-9813.

5. Sayama, K., et al. 2001. Apoptosis signal-regulating kinase 1 (ASK1) is an intracellular inducer of keratinocyte differentiation. J. Biol. Chem 276:999-1004.

6. Liu, Y., Yin, G., Surapisitchat, J., Berk, B.C., and Min, W. 2001. Laminar flow inhibits TNF-induced ASK1 activation by preventing dissociation of ASK1 from its inhibitor 14-3-3. J. Clin. Invest. 107:917-923.

7. Liu, Y., and Min, W. 2002. Thioredoxin promotes ASK1 ubiquitination and degradation to inhibit ASK1-mediated apoptosis in a redox activity-independent manner. Circ. Res. 90:1259-1266.

8. Tobiume, K., et al. 2001. ASK1 is required for sustained activations of JNK/p38 MAP kinases and apoptosis. EMBO Rep. 2:222-228

9. Nishitoh, H., et al. 1998. ASK1 is essential for JNK/SAPK activation by TRAF2. Mol. Cell. 2:389-395.

10. Saitoh, M., et al. 1998. Mammalian thioredoxin is a direct inhibitor of apoptosis signal-regulating kinase (ASK) 1. EMBO J. 17:2596-2606.

11. Gotoh, Y., and Cooper, J.A. 1998. Reactive oxygen species- and dimerization-induced activation of apoptosis signal-regulating kinase 1 in tumor necrosis factor-alpha signal transduction. J. Biol. Chem. 273:17477-17482.

12. Liu, H., Nishitoh, H., Ichijo, H., and Kyriakis, J.M. 2000. Activation of apoptosis signal-regulating kinase 1 (ASK1) by tumor necrosis factor receptor-associated factor 2 requires prior dissociation of the ASK1 inhibitor thioredoxin. Mol. Cell. Biol. 20:2198-2208.

13. Zhang, L., Chen, J., and Fu, H. 1999. Suppression of apoptosis signal-regulating kinase 1 -induced cell death by 14-3-3 proteins. Proc. Natl. Acad. Sci.U.S. A. 96:8511-8515.

14. Chen, H., Pong, R.C., Wang, Z., and Hsieh, J.T. 2002. Differential regulation of the human gene DAB2IP in normal and malignant prostatic epithelia: cloning and characterization. Genomics. 79:573-581.

15. Wang, Z., et al. 2002. The mechanism of growth-inhibitory effect of DOC-2/DAB2 in prostate cancer. Characterization of a novel GTPaseactivating protein associated with $\mathrm{N}$-terminal domain of DOC-2/DAB2 J. Biol. Chem. 277:12622-12631.
16. Min, W., et al. 1998. The N-terminal domains target TNF receptor-associated factor- 2 to the nucleus and display transcriptional regulatory activity. J. Immunol. 161:319-324.

17. Paddison, P.J., Caudy, A.A., Bernstein, E., Hannon, G.J., and Conklin, D.S. 2002. Short hairpin RNAs (shRNAs) induce sequence-specific silencing in mammalian cells. Genes Dev. 16:948-958.

18. Yin, G., et al. 2002. Endostatin gene transfer inhibits joint angiogenesis and pannus formation in inflammatory arthritis. Mol. Ther. 5:547-554.

19. Rizo, J., and Sudhof, T.C. 1998. C2-domains, structure and function of a universal Ca2+-binding domain. J. Biol. Chem. 273:15879-15882.

20. Pan, S., et al. 2002. Etk/Bmx as a tumor necrosis factor receptor type 2specific kinase: role in endothelial cell migration and angiogenesis. Mol. Cell. Biol. 22:7512-7523.

21. Surapisitchat, J., et al. 2001. Fluid shear stress inhibits TNF-alpha activation of JNK but not ERK1/2 or p38 in human umbilical vein endothelial cells: inhibitory crosstalk among MAPK family members. Proc. Natl. Acad. Sci. U. S. A. 98:6476-6481.

22. Zou, X., et al. 2001. The cell cycle-regulatory CDC25A phosphatase inhibits apoptosis signal-regulating kinase 1. Mol. Cell. Biol. 21:4818-4828

23. Morita, K., et al. 2001. Negative feedback regulation of ASK1 by protein phosphatase 5 (PP5) in response to oxidative stress. EMBO J. 20:6028-6036

24. Song, J.J., et al. 2002. Role of glutaredoxin in metabolic oxidative stress. Glutaredoxin as a sensor of oxidative stress mediated by H2O2. J. Biol. Chem. 277:46566-46575.

25. Dorion, S., Lambert, H., and Landry, J. 2002. Activation of the p38 signaling pathway by heat shock involves the dissociation of glutathione S-transferase Mu from Ask1. J. Biol. Chem. 277:30792-30797.

26. Park, H.S., et al. 2002. Heat shock protein hsp72 is a negative regulator of apoptosis signal-regulating kinase 1. Mol. Cell. Biol. 22:7721-7730.

27. Tobiume, K., Saitoh, M., and Ichijo, H. 2002. Activation of apoptosis signal-regulating kinase 1 by the stress-induced activating phosphorylation of pre-formed oligomer. J. Cell. Physiol. 191:95-104.

28. McDonald, P.H., et al. 2000. Beta-arrestin 2: a receptor-regulated MAPK scaffold for the activation of JNK3. Science. 290:1574-1577.

29. Matsuura, H., et al. 2002. Phosphorylation-dependent scaffolding role of JSAP1/JIP3 in the ASK1-JNK signaling pathway. A new mode of regulation of the MAP kinase cascade. J. Biol. Chem. 277:40703-40709.

30. Zama, T., et al. 2002. Scaffold role of a mitogen-activated protein kinase phosphatase, SKRP1, for the JNK signaling pathway. J. Biol. Chem. 277:23919-23926. 